\title{
B-mode ultrasound diagnostic flowchart for solid breast masses: JABTS BC-01 study
}

\author{
Takanori Watanabe ${ }^{1}$ (C) Takuhiro Yamaguchi $^{2} \cdot$ Eriko Tohno $^{3} \cdot$ Hiroko Tsunoda $^{4} \cdot$ Setsuko Kaoku $^{5} \cdot$ Kanako Ban $^{6}$. \\ Ryoji Watanabe $^{7} \cdot$ Takeshi Umemoto $^{8} \cdot$ Koichi Hirokaga $^{9} \cdot$ Toshikazu Ito $^{10}$
}

Received: 18 July 2020 / Accepted: 6 November 2020 / Published online: 3 January 2021

(c) The Author(s) 2021

\begin{abstract}
Purpose Breast ultrasound has been widely used as an essential examination for diagnosing breast cancer. However, standardized diagnostic criteria are as yet lacking. This study aimed to develop a simple diagnostic flowchart for beginners learning breast ultrasonography. The diagnostic flowchart was developed based on the recall criteria widely used in Japan.

Methods We conducted a multicenter study to examine recall criteria usefulness in the diagnostic phase of breast disease. Women with ultrasound-visible breast masses who underwent B-mode breast ultrasound examination were recruited from 22 hospitals in Japan between September 2009 and January 2010. B-mode images were evaluated by members of the centralized image interpretation committee. We developed the new diagnostic flowchart based on the results. The usefulness of the diagnostic flowchart was assessed by employing datasets from the current study and another study which we conducted (BC-04 study).

Results We evaluated 1045 solid masses (malignant: 495, benign: 550). Multivariate analysis showed that shape, margin, echogenic halo, interruption of the mammary gland interface, and depth width ratio were significant findings for distinguishing between benign and malignant masses. We modified the recall criteria and developed our novel diagnostic flowchart using these findings. The sensitivity and specificity of the new flowchart (current study: 0.97, 0.45; BC-04 study dataset: $0.95,0.45$ ) were similar to those of experts (current study: 0.96, 0.54; BC-04 study dataset: 0.98, 0.38).

Conclusion We developed a simple diagnostic flowchart for breast ultrasound. This flowchart is anticipated to be applicable to educating beginners learning breast ultrasound.
\end{abstract}

Keywords Breast ultrasound $\cdot$ Breast cancer $\cdot$ Diagnostic criteria $\cdot$ Multivariate analysis $\cdot$ Multicenter study

Takanori Watanabe

tak.mac@mac.com

1 Department of Breast Surgery, National Hospital Organization Sendai Medical Center, 2-11-12 Miyaginohara, Miyagino-ku, Sendai, Miyagi 983-8520, Japan

2 Division of Biostatistics, Tohoku University Graduate School of Medicine, 1-1 Seiryo-machi, Aoba-ku, Sendai, Miyagi 980-8574, Japan

3 Tsukuba International Breast Clinic, 2F Tsukuba CITYIA Bldg., 2-8-8 Azuma, Tsukuba, Ibaraki 305-0031, Japan

4 Department of Radiology Diagnostic Breast Imaging, St Luke's International Hospital, 9-1 Akashi-cho, Chuo-ku, Tokyo 104-8560, Japan

5 Department of Ultrasonics, National Hospital Organization Osaka National Hospital, 2-1-14 Hoenzaka, Chuo-ku, Osaka, Osaka 540-0006, Japan
6 Department of Cancer Detection and Diagnosis, Tokyo Health Service Association, 1-2 Ichigaya-sadohara-cho, Shinjuku-ku, Tokyo 162-8402, Japan

7 Department of Breast Center, Itoshima Medical Association Hospital, 532-1 Urashi, Itoshima, Fukuoka 819-1112, Japan

8 Department of Senology, Moriya Keiyu Hospital, 980-1 Tatsuzawa, Moriya, Ibaraki 302-0118, Japan

9 Department of Breast Surgery, Hyogo Cancer Center, 13-70 Kitaoji, Akashi, Hyogo 673-8558, Japan

10 Department of Surgery, Faculty of Medicine, Kindai University, 377-2 Ohno-Higashi, Osaka-Sayama, Osaka 589-8511, Japan 


\section{Introduction}

Breast ultrasound has improved remarkably due to advances in imaging technologies, such as tissue harmonic imaging, spatial compounding, Doppler ultrasound, and elastography. These advances have improved the ability to make an accurate differential diagnosis between benign and malignant lesions. However, diagnostic criteria for breast masses have yet to be standardized.

Breast ultrasound and mammography have been widely used as essential examinations for diagnosing breast cancer in Japan. The Japan Association of Breast and Thyroid Sonology (JABTS) was established in 1998. In 2004, JABTS published the Guidelines for Breast Ultrasound Diagnosis (1st edition) [1]. In the Guidelines, we proposed a diagnostic flowchart for breast masses using the recall criteria for ultrasound breast cancer screening. This flowchart and the criteria were developed based on the opinions of breast ultrasound experts. The recall criteria are very simple, and were developed for breast ultrasound screening. The diagnostic flowchart was developed for breast ultrasound diagnosis. However, the diagnostic flowchart was complex and difficult to remember. As a result, the diagnostic flowchart is not now in widespread use in Japan, although the recall criteria are widely used. There is, however, a problem with using the recall criteria for diagnosing breast masses. Since biopsy is performed to confirm the final diagnosis of breast cancer, it is important to decide whether to recommend a biopsy or observation at the time of breast ultrasound diagnosis. The recall criteria are not applicable to making this judgment. Therefore, we cannot use the recall criteria for this purpose. The aim of this study was to develop a simple new diagnostic flowchart for solid breast masses to facilitate the decision as to whether biopsy or observation should be recommended. We conducted a multicenter study and obtained findings useful for distinguishing between benign and malignant masses. Based on the results, we developed the novel diagnostic flowchart presented herein. To evaluate the usefulness of this new diagnostic flowchart, we employed a patient dataset from another of our multicenter studies in addition to the data obtained in this study.

\section{Materials and methods}

\section{Data collection}

Women with an ultrasound-visible breast mass who underwent B-mode breast ultrasound examination were recruited from 22 hospitals in Japan between September
2009 and January 2010. Ultrasound units with linear transducers exceeding $10 \mathrm{MHz}$ were used in this study. Exclusion criteria were as follows: 1 . simple cysts, 2 . lesions already being followed by ultrasound, 3 . lesions subjected to vacuum-assisted biopsy at another hospital, 4. masses larger than $5 \mathrm{~cm}$ in maximum diameter. Biopsy or observation was selected according to the routine clinical practices of each hospital. Lesions with no significant change during the 2 years of observation were regarded as being benign in this study. Static B-mode digital images and histopathological data without personal information were collected at the clinical research data center at Tohoku University Hospital.

\section{Informed consent}

The institutional review board or the ethics committee at each hospital approved this prospective observational study. Written informed consent was not required in this trial according to the ethical guidelines for epidemiological research in Japan [2]. There are two reasons for this. First, this trial did not use human biological specimens. Second, B-mode ultrasound is conducted as a routine examination for breast cancer diagnosis. However, public disclosure of information obtained in this study is required by all participating hospitals. When a patient refused to allow use of their clinical data, their data were not used.

\section{Centralized image interpretation committee}

Static B-mode digital images were evaluated by members of the centralized image interpretation committee comprised of 26 specialists with no knowledge of the clinical information (except for age) or the histopathological data. These 26 breast ultrasound specialists working in Japan included three radiologists, 19 breast surgeons, and four ultrasonographers. All were members of the Terminology and Diagnostic Criteria Committee of the JABTS. The 26 ultrasound specialists were divided into 13 pairs. Pairs of specialists evaluated each of the ultrasound images. If interpretation was difficult, the images were discussed by all members of the committee. The quality of liquid crystal image displays used for the centralized image interpretation was confirmed by TG18-QC pattern (American Association of Physicists in Medicine) [3]. The ultrasound findings and categories of each mass were reported by the centralized image interpretation committee. After evaluation of findings, such as shape, margin (Fig. 1a), internal echoes, posterior echoes, depth/width ratio (DW ratio, Fig. 1b), echogenic halo (echogenic rim, Fig. 1c), and interruption of the mammary gland interface (Fig. 1d), the B-mode category was determined by consensus. 
Fig. 1 a Margin, b depth width ratio (DW ratio), c echogenic halo (echogenic rim), d interruption of mammary gland interface
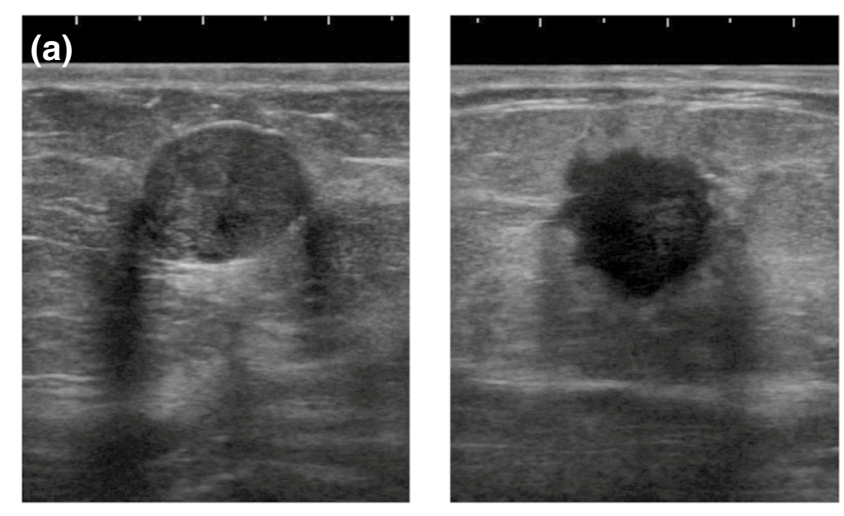

Well defined and smooth (Circumscribed)

Well defined and rough

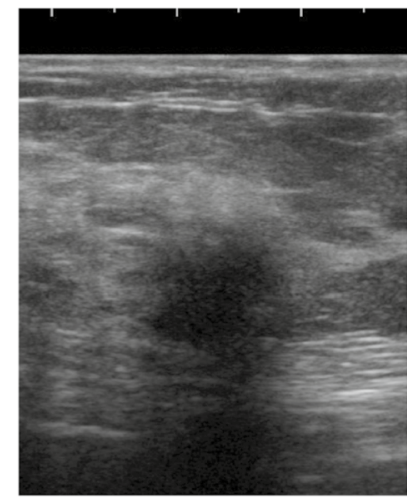

Indistinct
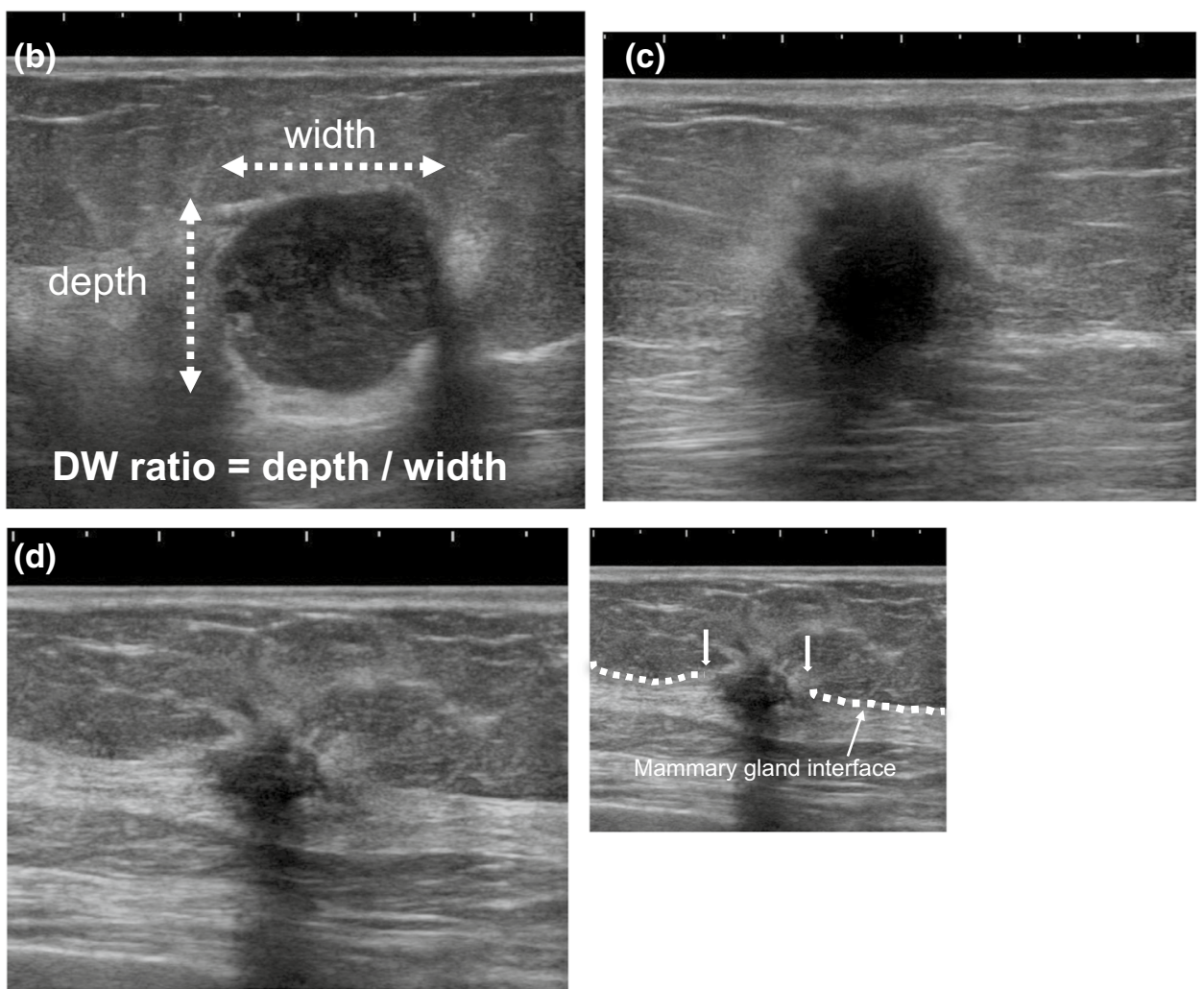

\section{Japanese category}

In Japan, we use Japanese categories; C1: normal, C2: benign, C3a: probably benign (observation is recommended), C3b: probably benign (biopsy is recommended), C4: suspicion of malignancy, C5: malignant [1]. Japanese categories differ from those of the Breast Imaging Reporting and Data System (BI-RADS) [4]. Japanese C3b corresponds to BI-RADS category 4A (biopsy is recommended) (Table 1).
Table 1 Correspondence between Japanese and BI-RADS categories

\begin{tabular}{ll}
\hline Japanese Category & $\begin{array}{l}\text { BI-RADS } \\
\text { Category }\end{array}$ \\
\hline 1 & 1 \\
2 & 2 \\
$3 \mathrm{a}$ & 3 \\
$3 \mathrm{~b}$ & $4 \mathrm{~A}$ \\
4 & $4 \mathrm{~B}, 4 \mathrm{C}$ \\
5 & 5 \\
\hline
\end{tabular}

$B I-R A D S$ breast imaging reporting and data system 


\section{The recall criteria for ultrasound breast cancer screening}

The recall criteria for ultrasound breast cancer screening include criteria pertaining to breast masses (simple cysts, complex cystic and solid masses) and breast non-mass lesions $[1,5]$. In this study, we focused only on solid breast masses, and we developed a new diagnostic flowchart for solid breast masses based on the recall criteria. Figure 2 shows the recall criteria pertaining to solid masses (2004 version) [6]. Herein, we show a slightly simplified version of the original recall criteria to enhance understanding. The criteria are divided into three sections. The first section categorizes obviously benign masses (fibroadenomas) as $\mathrm{C} 2$. Typical fibroadenomas have an oval shape, circumscribed margin, diameter less than $2 \mathrm{~cm}$, and a very low DW ratio. In this study, we defined "very low DW ratio" as less than 0.5 . Typical calcified fibroadenomas are characterized by coarse calcifications. The second section categorizes obviously malignant or highly suspicious masses as C4 or C5. Typical malignant masses show an echogenic halo and/or interruption of the mammary gland interface [7]. Masses with a high possibility of malignancy show echogenic foci within the mass. In the third section, the remaining masses are categorized as $\mathrm{C} 2,3$, or 4 according to the size and DW ratio. Since the third section of the recall criteria does not distinguish between C3a (observation) and C3b (biopsy), it cannot serve to determine whether biopsy or observation should be recommended. In 2014, an item pertaining to complicated cysts was added to the first section of the recall criteria [1,5]. However, since the B-mode images were evaluated before 2014, this item was not included in the present study.

\section{Statistical analysis}

Data collection and statistical analyses were conducted by the Clinical Research Data Center of Tohoku University Hospital. Statistical analyses were conducted using SAS Version 9.4 (SAS Institute, Inc., Cary, NC, USA). Univariate analysis was conducted using chi-square tests. Multivariate analysis was conducted using logistic regression.

\section{New diagnostic flowchart for solid masses}

Based on the results of the statistical analysis, the Terminology and Diagnostic Criteria Committee of the JABTS endeavored to develop a new diagnostic flowchart. To facilitate user recollection of the essential points, we developed this new diagnostic flowchart based on the recall criteria already widely used in Japan.

\section{Verification of the usefulness of the new diagnostic flowchart for solid masses}

The usefulness of our novel diagnostic flowchart for solid masses was evaluated by comparing the sensitivity and specificity determined by experts with those determined based on the new diagnostic flowchart.
Fig. 2 The recall criteria for solid masses (2004 version)

\section{Solid masses}

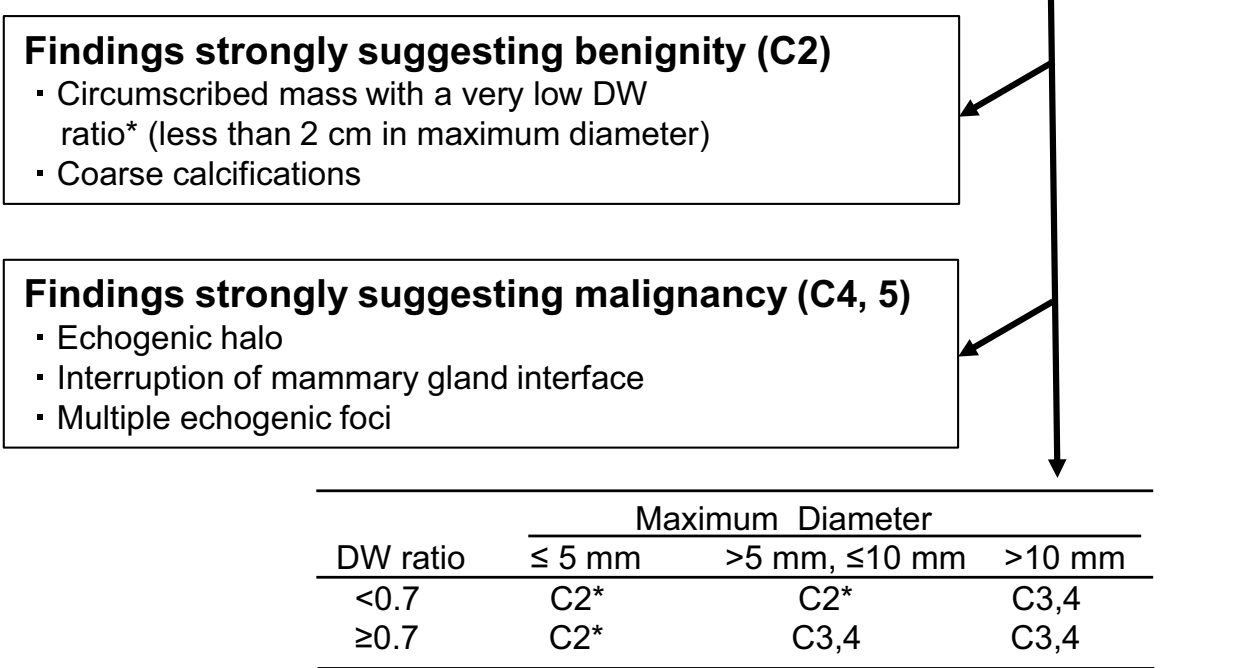

* If the shape is irregular, category 3 or higher is applicable. 
We emphasize the importance of using a dataset from a different patient population, in addition to the one obtained in this study, to allow comparative evaluation of the usefulness of the new diagnostic flowchart. Therefore, in addition to data from the current study (JABTS BC-01), we employed the patient dataset from our JABTS BC-04 study [8]. The JABTS BC-04 study aimed to develop diagnostic criteria for color Doppler examination of solid masses in the breast, and was conducted from 2013 to 2017 . The dataset from the JABTS BC-04 study included 839 malignant masses and 569 benign masses.

The datasets from the current (JABTS BC-01) study and the JABTS BC-04 study include findings and categories of solid masses determined by the centralized image interpretation committee. Categories of the new diagnostic flowchart were mechanically converted using the findings contained in the datasets. As a result, there were two categories for each mass; one determined by a specialist and the other based on the new diagnostic flowchart. We calculated and then compared sensitivity and specificity using these two categories. For statistical analyses of sensitivity and specificity, Japanese categories 2 and $3 \mathrm{a}$ (only observation is recommended) were considered to be negative, while categories $3 b, 4$, and 5 (biopsy is recommended) were taken to be positive.

\section{Study registration}

The JABTS BC-01 study is registered with the University Hospital Medical Information Network, Japan (No. UMIN000007603).

Fig. 3 Flow diagram of registered masses

\section{Results}

Between September 2009 and January 2010, 1412 ultrasound-visible breast masses were registered from 22 hospitals. Final enquiries regarding the histopathology and clinical observations were conducted in March 2014. Of the 1412 masses, six were excluded due to patient withdrawal, four due to missing data, two due to being simple cysts, and 18 due to being unevaluable by the centralized image interpretation committee because of inadequate image quality. Three hundred and five (55.3\%) of 551 observational masses lacked 2-year observation results. Of the remaining 1077 masses, 1045 were solid. Since the number of mixed masses was only 32, we evaluated the 1045 solid masses (malignant: 495 (468 patients), benign: 550 (459 patients)) in this study (Fig. 3). Mean sizes of malignant and benign masses were $1.6 \pm 0.78 \mathrm{~cm}(0.3-4.4)$ and $1.2 \pm 0.71 \mathrm{~cm}(0.3-5.5)$, respectively. The ages of the 468 patients with malignant mases and the 459 patients with benign masses were $56.8 \pm 12.5$ years (mean \pm standard deviation, range: $30-95)$ and $45.1 \pm 11.9$ years (13-76), respectively. The histopathological results of the 1045 masses are shown in Table 2. Biopsy was performed for 799 masses, of which 495 were malignant and 304 were benign. Invasive carcinoma of no special type accounted for $80 \%$ of malignant masses and ductal carcinoma in situ accounted for $10 \%$.

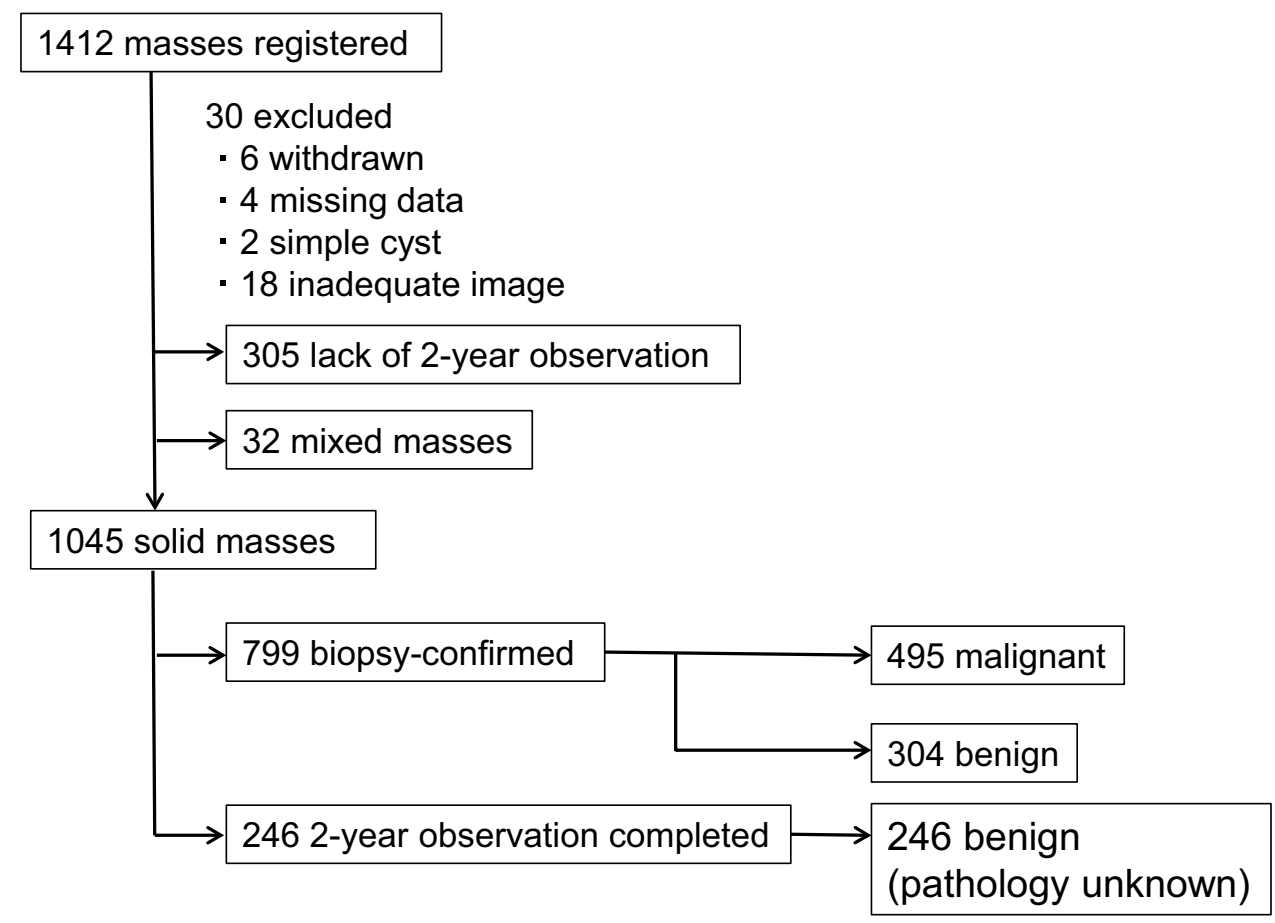


Table 2 Histopathological results $(n=1045)$

\begin{tabular}{ll}
\hline Malignant $(n=495)$ & \\
Ductal carcinoma in situ & 47 \\
Invasive carcinoma of no special type & 402 \\
Invasive lobular carcinoma & 20 \\
Mucinous carcinoma & 18 \\
Carcinoma with apocrine differentiation & 2 \\
Tubular carcinoma & 2 \\
Other (malignant) & 4 \\
Benign (n=550) & \\
Fibroadenoma & 115 \\
Fibrocystic disease & 71 \\
Intraductal papilloma & 28 \\
Complicated cyst & 25 \\
Phyllodes tumor & 8 \\
Fibrosis & 7 \\
Ductal hyperplasia & 5 \\
Tubular adenoma & 2 \\
Other (benign) & 43 \\
Unknown (benign) $^{\mathrm{a}}$ & 246 \\
\hline a2-year observation completed &
\end{tabular}

a2-year observation completed

Table 3 Malignancy rates in lesions found to be benign or malignant based on JABTS Recall criteria

\begin{tabular}{ll}
\hline Findings & Malignancy rate \\
\hline $\begin{array}{l}\text { Obvious benign findings (C2) } \\
\text { Circumscribed mass with a very low DW ratio }\end{array}$ & $1.6 \%(1 / 62)$ \\
$\quad($ diameter less than $2 \mathrm{~cm})$ & $11.1 \%(1 / 9)$ \\
$\quad$ Coarse calcification & \\
Obvious malignant findings $(\mathrm{C} 4,5)$ & $97.5 \%(153 / 157)$ \\
$\quad$ Echogenic halo & $90.8 \%(246 / 271)$ \\
Interruption of mammary gland interface & $59.3 \%(32 / 54)$ \\
Multiple echogenic foci &
\end{tabular}

\section{Utility of the recall criteria as a diagnostic flowchart}

We evaluated the usefulness of the recall criteria as a diagnostic flowchart. In the first and second sections of the recall criteria, $12.5 \%$ (69/550) of benign masses and 87.1\% (431/495) of malignant masses were detected. Table 3 shows the malignancy rate in the first and second sections of the recall criteria. Regarding circumscribed masses with a very low DW ratio (diameter less than $2 \mathrm{~cm}$ ), 98.4\% (61/62) were benign, and $89 \%(8 / 9)$ of masses with coarse calcifications were benign. Furthermore, $97.5 \%$ (153/157) of masses with an echogenic halo, $90.8 \%$ (246/271) showing interruption of the mammary gland interface, and $59.3 \%$ (32/54) with multiple echogenic foci were malignant. Since the third section of the recall criteria for solid masses cannot be used in a diagnostic flowchart, we did not include it in the present evaluation.

\section{Frequencies of malignant and benign masses according to the ultrasound findings (Table 4)}

As to shape, $89.5 \%$ of oval masses were benign, and $76.0 \%$ of irregular masses were malignant. Regarding the DW ratio, $66.6 \%$ of the masses with a DW ratio less than 0.7 were benign, and $67.4 \%$ of those with a DW ratio of at least 0.7 were malignant. As to the margin, $91.1 \%$ of circumscribed masses were benign, and $75.5 \%$ with indistinct margins were malignant. Furthermore, $97.5 \%$ of the masses with an echogenic halo and $90.8 \%$ of those showing interruption of the mammary gland interface were malignant. Regarding echogenic foci, $75.3 \%$ of the masses with echogenic foci were malignant.

\section{Multivariate analysis}

Multivariate analysis showed that shape, DW ratio, margin, echogenic halo, and interruption of the mammary gland interface were significant findings for distinguishing between benign and malignant masses (Table 5).

\section{The new diagnostic flowchart for solid masses} (Fig. 4)

Using these results, the Terminology and Diagnostic Criteria Committee of the JABTS discussed and proposed a new diagnostic flowchart based on the recall criteria. Since the first and second sections of the recall criteria were demonstrated to be very useful, we applied them as the first and second sections of the new diagnostic flowchart. Then, we developed the third section of the new diagnostic flowchart. Of the five significant findings for distinguishing between benign and malignant masses by multivariate analysis, echogenic halo and interruption of the mammary gland interface were included in the second section. Therefore, we used shape, margin, and DW ratio in the third section. Among these parameters, those raising suspicion of malignancy were irregular shape, well-defined and rough/indistinct margin, and DW ratio $\geq 0.7$ (Fig. 4). If none of these three suspicious findings is present, the category is determined to be $3 \mathrm{a}$, and if at least one is present, the category is $3 \mathrm{~b}$. The committee proposed a new diagnostic flowchart for solid masses in May of 2015.

\section{Sensitivity and specificity of the new diagnostic flowchart}

We calculated sensitivity and specificity using the datasets from the current study (JABTS BC-01) and the JABTS BC-04 study. Details of the BC-04 study dataset are shown 
Table 4 Frequency of malignant and benign masses according to US findings $(n=1045)$

\begin{tabular}{|c|c|c|c|c|c|}
\hline Findings & Number of cases & Malignant & Benign & $\%$ Malignant & $\%$ Benign \\
\hline \multicolumn{6}{|l|}{ Shape } \\
\hline Oval & 313 & 33 & 280 & $10.5 \%$ & $89.5 \%$ \\
\hline Round & 56 & 25 & 31 & $44.6 \%$ & $55.4 \%$ \\
\hline Lobulated & 220 & 104 & 116 & $47.3 \%$ & $52.7 \%$ \\
\hline ( 2 or 3 undulations) & 141 & 50 & -91 & $35.5 \%$ & $64.5 \%$ \\
\hline (More than 4 undulations) & 73 & 48 & -25 & $65.8 \%$ & $34.2 \%$ \\
\hline Polygonal & 47 & 22 & 25 & $46.8 \%$ & $53.2 \%$ \\
\hline Irregular & 409 & 311 & 98 & $76.0 \%$ & $24.0 \%$ \\
\hline \multicolumn{6}{|l|}{ DW ratio } \\
\hline$<0.7$ & 616 & 206 & 410 & $33.4 \%$ & $66.6 \%$ \\
\hline$(<0.5)$ & (216) & $(50)$ & $(166)$ & $(23.1 \%)$ & $(76.9 \%)$ \\
\hline$(0.5-0.7)$ & $(400)$ & $(156)$ & $(244)$ & $(39.0 \%)$ & $(61.0 \%)$ \\
\hline$\geq 0.7$ & 429 & 289 & 140 & $67.4 \%$ & $32.6 \%$ \\
\hline \multicolumn{6}{|l|}{ Margin } \\
\hline Circumscribed & 337 & 30 & 307 & $8.9 \%$ & $91.1 \%$ \\
\hline Well-defined and rough & 486 & 298 & 188 & $61.3 \%$ & $38.7 \%$ \\
\hline Indistinct & 220 & 166 & 54 & $75.5 \%$ & $24.5 \%$ \\
\hline Obscure & 2 & 1 & 1 & $50.0 \%$ & $50.0 \%$ \\
\hline \multicolumn{6}{|l|}{ Echogenic halo } \\
\hline Present & 157 & 153 & 4 & $97.5 \%$ & $2.5 \%$ \\
\hline Absent & 888 & 342 & 546 & $38.5 \%$ & $61.5 \%$ \\
\hline \multicolumn{6}{|l|}{ Internal echoes } \\
\hline \multicolumn{6}{|l|}{ Homogeneity } \\
\hline Homogeneous & 408 & 104 & 304 & $25.5 \%$ & $74.5 \%$ \\
\hline Heterogeneous & 637 & 391 & 246 & $61.4 \%$ & $38.6 \%$ \\
\hline \multicolumn{6}{|l|}{ Echo level } \\
\hline Anechoic & 0 & 0 & 0 & $0.0 \%$ & $0.0 \%$ \\
\hline Hypoechoic & 920 & 461 & 459 & $50.1 \%$ & $49.9 \%$ \\
\hline Isoechoic & 119 & 31 & 88 & $26.1 \%$ & $73.9 \%$ \\
\hline Hyperechoic & 6 & 3 & 3 & $50.0 \%$ & $50.0 \%$ \\
\hline \multicolumn{6}{|l|}{ Echogenic foci } \\
\hline Present & 97 & 73 & 24 & $75.3 \%$ & $24.7 \%$ \\
\hline Absent & 948 & 422 & 526 & $44.5 \%$ & $55.5 \%$ \\
\hline \multicolumn{6}{|l|}{ Coarse calcifications } \\
\hline Present & 9 & 1 & 8 & $11.1 \%$ & $88.9 \%$ \\
\hline Absent & 1036 & 494 & 542 & $47.7 \%$ & $52.3 \%$ \\
\hline \multicolumn{6}{|l|}{ Posterior echoes } \\
\hline Accentuating & 323 & 148 & 175 & $45.8 \%$ & $54.2 \%$ \\
\hline Not changing & 551 & 214 & 337 & $38.8 \%$ & $61.2 \%$ \\
\hline Attenuating & 168 & 132 & 36 & $78.6 \%$ & $21.4 \%$ \\
\hline Shadowing & 3 & 1 & 2 & $33.3 \%$ & $66.7 \%$ \\
\hline \multicolumn{6}{|c|}{ Interruption of mammary gland interface } \\
\hline Present & 271 & 246 & 25 & $90.8 \%$ & $9.2 \%$ \\
\hline Absent & 774 & 249 & 525 & $32.2 \%$ & $67.8 \%$ \\
\hline
\end{tabular}

in Table 6. With the current study dataset, sensitivity and specificity of the diagnostic flowchart were 0.97 and 0.45 , respectively. The sensitivity and specificity based on the evaluations performed by the specialists were 0.96 and 0.54 , respectively (Table 7). When we used the BC-04 study dataset, the respective sensitivity and specificity of the diagnostic flowchart were 0.95 and 0.45 , while the corresponding sensitivity and specificity for the specialists were 0.98 and 0.38 . 
Table 5 Multivariate analysis of B-mode features and benign/ malignant differential diagnosis (logistic regression)

\begin{tabular}{|c|c|c|c|c|}
\hline \multirow{2}{*}{$\begin{array}{l}\text { Findings } \\
\text { Shape }\end{array}$} & \multirow[t]{2}{*}{$\begin{array}{l}\text { Adjusted odds } \\
\text { ratio }\end{array}$} & \multicolumn{2}{|c|}{ 95\% Confidence interval } & \multirow[t]{2}{*}{$p$} \\
\hline & & & & \\
\hline Round vs. oval & 3.34 & 1.41 & 7.93 & 0.0062 \\
\hline Polygonal vs. oval & 2.04 & 0.79 & 5.29 & 0.141 \\
\hline Irregular vs. oval & 4.07 & 2.16 & 7.65 & $<.0001$ \\
\hline Lobulated vs. oval & 2.82 & 1.52 & 5.25 & 0.001 \\
\hline \multicolumn{5}{|l|}{ DW ratio } \\
\hline$\geq 0.7$ vs $<0.7$ & 2.64 & 1.7 & 4.1 & $<.0001$ \\
\hline \multicolumn{5}{|l|}{ Margin } \\
\hline Indistinct vs. circumscribed & 3.38 & 1.62 & 7.04 & 0.0012 \\
\hline Well-defined and rough vs. circumscribed & 5.3 & 3 & 9.39 & $<.0001$ \\
\hline \multicolumn{5}{|l|}{ Echogenic halo } \\
\hline Present vs. absent & 9.82 & 2.79 & 34.57 & 0.0004 \\
\hline \multicolumn{5}{|l|}{ Internal echoes } \\
\hline Heterogeneous vs. homogeneous & 1.18 & 0.74 & 1.86 & 0.49 \\
\hline \multicolumn{5}{|l|}{ Echo level } \\
\hline Severely hypoechoic vs. hypoechoic & 0.6 & 0.15 & 2.39 & 0.47 \\
\hline Isoechoic vs. hypoechoic & 0.58 & 0.3 & 1.12 & 0.1 \\
\hline Hyperechoic vs. hypoechoic & 1.18 & 0.07 & 18.89 & 0.91 \\
\hline \multicolumn{5}{|l|}{ Echogenic foci or coarse calcification } \\
\hline Echogenic foci vs. absent & 1.74 & 0.89 & 3.42 & 0.11 \\
\hline Coarse calcification vs absent & 0.18 & 0.03 & 1.11 & 0.06 \\
\hline \multicolumn{5}{|l|}{ Posterior echoes } \\
\hline Accentuating vs. not changing & 1.37 & 0.88 & 2.16 & 0.17 \\
\hline Attenuating vs. not changing & 0.98 & 0.52 & 1.87 & 0.96 \\
\hline Shadowing vs. not changing & 2.5 & 0.06 & 114.43 & 0.64 \\
\hline \multicolumn{5}{|l|}{ Interruption of mammary gland interface } \\
\hline Present vs. absent & 3.1 & 1.72 & 5.57 & 0.0002 \\
\hline
\end{tabular}

Fig. 4 New diagnostic flowchart for solid masses

\section{Solid masses}

Findings strongly suggesting benignity (C2)

- Circumscribed mass with a very low DW ratio (diameter less than $2 \mathrm{~cm}$ )

- Coarse calcifications

Findings strongly suggesting malignancy $(\mathrm{C} 4,5)$

- Echogenic halo

- Interruption of mammary gland interface

- Multiple echogenic foci

\begin{tabular}{lcc}
\hline \multicolumn{1}{c}{ suspicious findings } & none & at least one \\
\hline Well-defined and rough/indistinct & & \\
DW ratio $\geq 0.7$ & C3a (Follow-up) & C3b (Biopsy) \\
Irregular shape & & \\
\hline
\end{tabular}


Table 6 BC-04 study dataset $(n=1408)$

\begin{tabular}{|c|c|}
\hline Number of masses & \\
\hline Malignant: 839 (818 patients) & \\
\hline Benign: 569 (547 patients) & \\
\hline Age (mean \pm standard deviation, range) & \\
\hline Malignant (818 patients): $57.7 \pm 13.7$ (25-96) & \\
\hline Benign (547 patients): $44.8 \pm 12.8$ (12-87) & \\
\hline Mean sizes & \\
\hline Malignant: $1.7 \pm 0.84 \mathrm{~cm}(0.4-4.8)$ & \\
\hline Benign: $1.5 \pm 0.92 \mathrm{~cm}(0.3-4.8)$ & \\
\hline Histopathological results & \\
\hline Malignant (839) & \\
\hline Ductal carcinoma in situ & 53 \\
\hline Invasive carcinoma of no special type & 716 \\
\hline Invasive lobular carcinoma & 29 \\
\hline Mucinous carcinoma & 22 \\
\hline Carcinoma with apocrine differentiation & 5 \\
\hline Tubular carcinoma & 1 \\
\hline Other (malignant) & 18 \\
\hline Benign (569) & \\
\hline Fibroadenoma & 179 \\
\hline Fibrocystic disease & 104 \\
\hline Intraductal papilloma & 43 \\
\hline Complicated cyst & 3 \\
\hline Phyllodes tumor & 29 \\
\hline Ductal hyperplasia & 6 \\
\hline Tubular adenoma & 1 \\
\hline Other (benign) & 29 \\
\hline Unknown (benign) $^{\mathrm{a}}$ & 175 \\
\hline
\end{tabular}

a2-year observation completed

Table 7 Sensitivity and specificity using new diagnostic flowchart vs experienced specialists $(\mathrm{C} 2,3 \mathrm{a}$ vs. $\mathrm{C} 3 \mathrm{~b}, 4,5)$

\begin{tabular}{llllll}
\hline & \multicolumn{2}{l}{ Datasets } & & \\
\cline { 2 - 3 } & $\begin{array}{l}\text { Current study (JABTS } \\
\text { BC-01) }\left(n=1045^{\mathrm{a}}\right)\end{array}$ & & \multicolumn{2}{l}{$\begin{array}{l}\text { JABTS BC-04 study } \\
\left(n=1408^{\mathrm{b}}\right)\end{array}$} \\
\cline { 2 - 3 } \cline { 5 - 6 } & Sensitivity & Specificity & & Sensitivity & Specificity \\
\hline $\begin{array}{l}\text { New diagnos- } \\
\text { tic flowchart }\end{array}$ & 0.97 & 0.45 & 0.95 & 0.45 \\
\begin{tabular}{l} 
Specialists \\
\hline
\end{tabular} & 0.96 & 0.54 & 0.98 & 0.38 \\
\hline
\end{tabular}

${ }^{a}$ Malignant: 495, benign: 550

balignant: 839, benign: 569

\section{Discussion}

Ultrasonography is very useful for diagnosing breast cancer. There has been significant progress in distinguishing between malignant and benign masses using ultrasound since the early 1990s [9-12]. Several sonographic features based on shape, margin, and echo texture have been proposed for the diagnosis of breast masses [10, 13, 14]. At present, the BI-RADS classification is widely used globally [4]. However, BI-RADS does not include ultrasound diagnostic criteria. Several studies have attempted to develop ultrasound diagnostic criteria $[15,16]$, but diagnostic criteria have yet to be standardized.

In Japan, JABTS developed a diagnostic flowchart for breast masses in 2004 [6]. The diagnostic flowchart was developed after 4 years of discussions among experts. However, the flowchart was complex and therefore did not come into widespread use in Japan. On the other hand, the recall criteria for ultrasound breast cancer screening are very simple and widely used in Japan for ultrasound screening of breast cancer [5]. It would be optimal if the recall criteria could be used as a diagnostic flowchart. However, the recall criteria cannot be used to decide whether to recommend a biopsy or observation, and thus cannot be applied in diagnostic flowchart form. Therefore, we needed to develop a novel diagnostic flowchart. To encourage widespread use in Japan, we aimed to simplify the new diagnostic flowchart and to apply the recall criteria already widely used in Japan.

The recall criteria were proposed by JABTS in 2004 [6], and a revised version was published in 2016 [5]. Clearly or typically benign (fibroadenoma) and malignant masses are identified by applying the first and second portions of the criteria. Several reports have described fibroadenoma findings [17-20]. These findings are oval shape, smooth and well-circumscribed margin, and low DW ratio. The DW ratio was reportedly less than 0.7 in $86 \%$ of fibroadenomas and, furthermore, fibroadenomas usually stopped growing at a size of 2-3 cm [18]. According to the recall criteria, typical fibroadenomas are defined as oval circumscribed masses less than $2 \mathrm{~cm}$ in diameter, with a very low DW ratio. In this study, $1.6 \%$ (1/62) of masses judged to be typical fibroadenomas were ultimately found to be malignant.

The two typically used malignant findings are echogenic halo and interruption of the mammary gland interface. Echogenic halo has been reported to have high predictive value for malignancy by multiple research groups [13, 14, 16]. Interruption of the mammary gland interface was originally proposed by Konishi [7] in 1988. This finding has been used in Japan since the late twentieth century [21]. Our present study showed that this finding had high predictive value for malignancy. However, the predictive value of interruption of the mammary gland interface was slightly lower (90.8\%) than that of echogenic halo (97.5\%). Applying a combination of these two findings resulted in approximately half of breast cancers being interpreted as malignant. Features typical of malignancy $(\mathrm{C} 4,5)$ and of fibroadenoma $(\mathrm{C} 2)$ in the recall criteria had high diagnostic utility (Table 2 ). Therefore, we 
decided to incorporate the first and second sections of the recall criteria into the new diagnostic flowchart.

We also examined the applicability of the third section of the new diagnostic flowchart. We used three findings raising suspicion of malignancy, which multivariate analysis had shown to be useful for distinguishing between benign and malignant breast masses. We advocate performing a biopsy if any of these three suspicious findings is identified in breast masses with neither clearly typical benign nor malignant findings. The sensitivity and specificity of the new diagnostic flowchart using the dataset from the current study were 0.97 and 0.45 , respectively. The corresponding sensitivity and specificity of the current study for the specialists (centralized image interpretation committee) were 0.96 and 0.54. Furthermore, we examined the usefulness of the new diagnostic flowchart using our dataset from the BC-04 study [8]. The sensitivity and specificity of the new diagnostic flowchart using the BC-04 study dataset were 0.95 and 0.45 , respectively. The sensitivity and specificity for the specialists (centralized image interpretation committee) examining the BC-04 study were 0.98 and 0.38 . The specificity of the new flowchart was thus slightly inferior to that of the experts, but the sensitivity was higher. These results indicate that the new diagnostic flowchart is applicable, at a minimum, to diagnostic flowchart use for beginners. This flowchart is just a first step for beginners learning breast ultrasound. As they gain experience, beginners can progress to the intermediate and more advanced skill levels. This flowchart may even serve as a gateway allowing beginners to become experts in performing diagnostic ultrasound examinations of the breast.

We anticipate that this flowchart will become more sophisticated with ongoing revisions. As an example, parameters, such as age, elastographic findings, and color Doppler imaging, could potentially be incorporated into the flowchart. We hope that this flowchart will be useful not only to medical specialists but also to patients.

\section{Limitation}

Our proposed diagnostic flowchart was developed based on expert-judged imaging findings data. This flowchart may not work well if the inter-observer agreement between the beginners and the experts in evaluating each finding is low. Therefore, there is a need for education for beginners using this flowchart.

\section{Conclusion}

In this study, we developed a simple diagnostic flowchart for B-mode breast ultrasound. This flowchart is anticipated to be applicable to educating beginners learning breast ultrasound.
Acknowledgements We thank all of the patients who consented to the use of their ultrasound images for this study. We also thank all participating investigators and their support staff for their contributions to this study.

\section{Compliance with ethical standards}

Conflict of interest The authors have no conflicts of interest to declare.

Ethical statements The institutional review board or the ethics committee at each hospital approved this study.

Open Access This article is licensed under a Creative Commons Attribution 4.0 International License, which permits use, sharing, adaptation, distribution and reproduction in any medium or format, as long as you give appropriate credit to the original author(s) and the source, provide a link to the Creative Commons licence, and indicate if changes were made. The images or other third party material in this article are included in the article's Creative Commons licence, unless indicated otherwise in a credit line to the material. If material is not included in the article's Creative Commons licence and your intended use is not permitted by statutory regulation or exceeds the permitted use, you will need to obtain permission directly from the copyright holder. To view a copy of this licence, visit http://creativecommons.org/licenses/by/4.0/.

\section{References}

1. Japan association of breast and thyroid sonology (JABTS). Guidelines for breast ultrasound: Management and diagnosis. 3rd edition Tokyo: Nankodo; 2014 [In Japanese].

2. Ministry of Health, Labour and Welfare, Japan. Ethical guidelines for epidemiological research. Japan 2012. https://www.niph.go.jp/ wadai/ekigakurinri/guidelines.pdf.

3. Samei E. Assessment of display performance for medical imaging systems: executive summary of AAPM TG 18 report. Med Phys. 2005;32:1205-25.

4. American College of Radiology. ACR BI-RADS atlas: Breast imaging reporting and data system. 5th ed. Reston, VA: Author; 2013.

5. Terminology and diagnostic criteria committee of Japan society of ultrasonic in medicine. Recall criteria for ultrasound breast cancer screening. J Med Ultrasonics. 2016;43:301-13.

6. Japan Association of Breast and Thyroid Sonology (JABTS). Guidelines for breast ultrasound: Management and diagnosis. Tokyo:Nankodo; 2004 [In Japanese].

7. Konishi Y, Ogata M, Kuroki T, Tocjiko H, Fujimoto T, Morimoro Y, et al. Assessment on Differential Diagnosis between Tumorforming Mastopathy on Ultrasonography and T1 Breast Cancer. Jpn J Med Ultrasonics. 1988;15:44-51.

8. Watanabe T, Kaoku S, Yamaguchi T, Izumori A, Konno S, Okuno T, et al. Multicenter prospective study of color Doppler ultrasound for breast masses: Utility of our color Doppler method. Ultrasound in Med Biol. 2019;45:1367-79.

9. Jackson VP, Reynolds HE, Hawes DR. Sonography of the breast. Semin Ultrasound CT MR. 1996;17:460-75.

10. Stavros AT, Thickman D, Rapp CL, Dennis MA, Parker SH, Sisney GA. Solid breast nodules: use of sonography to distinguish between benign and malignant lesions. Radiology. 1995;196:123-34.

11. Jackson VP. Management of solid breast nodules: what is the role of sonography? Radiology. 1995;196:14-5. 
12. Paulinelli RR, Freitas-Junior R, Moreira MA, Moraes VA, Bernardes-Júnior JR, Vidal CS, et al. Risk of malignancy in solid breast nodules according to their sonographic features. J ultrasound Med. 2005;24:635-41.

13. Stavros AT. Breast ultrasound. In: Cynthia L. Rapp, Steve H. Parker, Editors. Philadelphia: Lippincott Williams \& Wilkins; 2004.

14. Helmut M. The practice of breast ultrasound: techniques, findings, differential diagnosis. New York: Thieme; 2000.

15. Costantini M, Belli P, Lombardi R, Franceschini G, Mulè A, Bonomo L. Characterization of solid breast masses -use of the sonographic breast imaging reporting and data system lexicon-. J Ultrasound Med. 2006;25:649-59.

16. Kim SJ, Ko EY, Shin JH, Kang SS, Mun SH, Han BK, et al. Application of sonographic BI-RADS to synchronous breast nodules detected in patients with breast cancer. Am J Roentgenol. 2008;191:653-8.

17. Fornage BD, Lorigan JG, Andry E. Fibroadenoma of the breast: sonographic appearance. Radiology. 1989;172:671-5.
18. Cole-Beuglet C, Soriano RZ, Kurts AB, Goldberg BB. Fibroadenoma of the breast: sonomammography correlated with pathology in 122 patients. AJR. 1983;140:369-75.

19. Jackson VP, Rothschild PA, Kreipke DL, Mail JT, Holden RW. The spectrum of sonpgraphic findings of fibroadenoma of the breast. Invest Radiol. 1986;21:34-40.

20. Venta LA, Dudiak CM, Salomon CG, Flisak ME. Sonographic evaluation of the breast. RadioGraphics. 1994;14:29-50.

21. Ueno E, Tohno E, Tsunoda H, Hirano M. Current status of ultrasound examination of the superficial organs (breast and thyroid). Rinsho Byori. 1993;41:15-25.

Publisher's Note Springer Nature remains neutral with regard to jurisdictional claims in published maps and institutional affiliations. 